\title{
Dynamics on Networks of Manifolds
}

\author{
Lee DEVILLE and Eugene LERMAN
}

Department of Mathematics, University of Illinois, USA

E-mail: rdeville@illinois.edu,lerman@illinois.edu

URL: http://www.math.illinois.edu/ rdeville/, http://www.math.illinois.edu/ lerman/

Received April 24, 2014, in final form February 24, 2015; Published online March 12, 2015

http://dx.doi.org/10.3842/SIGMA.2015.022

\begin{abstract}
We propose a precise definition of a continuous time dynamical system made up of interacting open subsystems. The interconnections of subsystems are coded by directed graphs. We prove that the appropriate maps of graphs called graph fibrations give rise to maps of dynamical systems. Consequently surjective graph fibrations give rise to invariant subsystems and injective graph fibrations give rise to projections of dynamical systems.
\end{abstract}

Key words: coupled cell networks; open dynamical systems; control systems; morphisms of dynamical systems

2010 Mathematics Subject Classification: 34C14; 18D99

\section{Introduction}

Given a dynamical system, one often starts by trying to find invariant subsystems; these include equilibria, periodic orbits, and higher dimensional invariant submanifolds. In addition, constructing projections onto smaller systems as well as conjugacies and semi-conjugacies with simpler systems are generally useful for understanding the qualitative properties of dynamical systems. All of these objects: invariant subsystems, projections, conjugacies and semiconjugacies can be realized as maps of dynamical systems (q.v. Definition 2.1). Thus the search for maps between dynamical systems may be considered one of the fundamental questions of the subject.

In this paper we give a precise definition of a continuous time dynamical system made up of interacting open subsystems. We then exploit the combinatorial aspect of such systems to produce maps of dynamical systems out of appropriate maps of graphs called graph fibrations (q.v. Definition 3.1). We show that in particular surjective graph fibrations give rise to invariant subsystems and injective graph fibrations give rise to projections of dynamical systems.

The present work is part of an ongoing project. In [5] we reformulated the groupoid formalism of Golubitsky, Pivato, Stewart and Török [7,8] for coupled cell networks (which are systems of ordinary differential equations) in a coordinate free manner and extended it to groupoidinvariant vector fields on manifolds. A preliminary version was posted as [4]. We later realized that groupoid invariance of vector fields is not needed for the existence of invariant subspaces. With the benefit of hindsight we see that the theory developed in [5] is an equivariant version of the theory that we develop here. We would like to point out that dropping groupoid invariance makes the theory much simpler and more flexible. In particular we expect the results of this paper to readily generalize to hybrid systems.

The absence of explicit groupoid symmetries makes our work close in spirit to the approach to dynamics on networks advocated by Field [6]. Unlike Field we find it convenient to use the language of category theory. We also find it useful to borrow the notions of open systems and their interconnection from engineering (see, for example $[3,9,12]$ ) and the definition of 
a graph fibration from computer science [2] (see [11] for a history of the notion and alternative terminologies).

We believe that both Field's approach and ours is based on the existence of a certain algebraic structure which at the present time is not completely understood. Open continuous time systems form an algebra over a certain operad [10]. This operad is implicit in the work of Field [6]. A piece of this algebra shows up in our work as the interconnection maps (see Theorem 2.32). We do not understand yet how graph fibrations interact with this operad and plan to address this issue in a future work.

The goal of this paper is to construct a category of networks of continuous time systems and a functor to the category of dynamical systems. A network in our sense consists of

- a finite directed graph $G$ with a set of nodes $G_{0}$,

- a phase space function $\mathcal{P}$ that assigns to each node of the graph an appropriate phase space (which we take to be a manifold),

- a family of open systems $\left\{w_{a}\right\}_{a \in G_{0}}$ (one for each node $a$ of the graph $G$ ) consistent in an appropriate way with the structure of the graph, and

- an interconnection map $\mathscr{I}$ that turns these open systems into a vector field on the product $\prod_{a \in G_{0}} \mathcal{P}(a)$ of the phase spaces of the nodes.

Our main result, Theorem 3.11, shows that graph fibrations compatible with phase space functions give rise to maps of dynamical systems. This allows us to define a functor from dynamical systems on networks to general dynamical systems.

The reader may wonder what motivates us to come up with these definitions and constructions. Indeed there are many different kinds of objects in engineering, science and mathematics that are called "networks". The notion of a network in the present paper arose from the following idea, which is implicit in the literature on coupled cell networks. Imagine a physical system modeled by a vector field $X$ on a manifold $M ; M$ is the collection of all possible states of the system. Such systems are common in classical mechanics, to give one example. Suppose further that our system consists of two interacting subsystems. We can model this by saying that the collection of states of the first subsystem forms a manifold $M_{1}$ and the second a manifold $M_{2}$. We would like the states of the big system to be completely determined by the states of its subsystems. We model this by requiring that $M=M_{1} \times M_{2}$. A vector field $X$ on $M_{1} \times M_{2}$ then has to be of the form

$$
X\left(x_{1}, x_{2}\right)=\left(X_{1}\left(x_{1}, x_{2}\right), X_{2}\left(x_{1}, x_{2}\right)\right),
$$

where

$$
X_{1}\left(x_{1}, x_{2}\right) \in T_{x_{1}} M_{1} \quad \text { for all } \quad\left(x_{1}, x_{2}\right) \in M_{1} \times M_{2},
$$

with a similar equation holding for $X_{2}: M_{1} \times M_{2} \rightarrow T M_{2}$. Note that the functions $X_{1}, X_{2}$ are not vector fields. They are open systems in the sense of Definition 2.22. Moreover the vector field $X$ may be considered to be a result of interconnecting $X_{1}$ and $X_{2}$ (see Proposition 2.24 and Theorem 2.32).

To continue with our example, observe that the evolution of the subsystem 1 depends on its state and the state of the subsystem 2. Similarly the evolution the second subsystem depends on its state and the state of the subsystem 1 . These mutual influences can be pictured graphically as

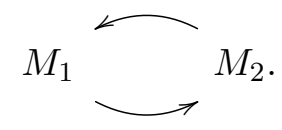


Assume now that the map $X_{2}$ does not really depend on the points of $M_{1}$. That is, there is a map $Y: M_{2} \rightarrow T M_{2}$ with $Y\left(x_{2}\right) \in T_{x_{2}} M_{2}$ and $X_{2}\left(x_{1}, x_{2}\right)=Y\left(x_{2}\right)$ for all $\left(x_{1}, x_{2}\right) \in M_{1} \times M_{2}$. We can picture this as

$$
M_{1} \longleftarrow M_{2}
$$

and say that the second subsystem drives the first but not conversely. This way of picturing a system made up of interacting subsystems generalizes to any number of subsystems. For example, a system may be made up of three interacting subsystems like this:

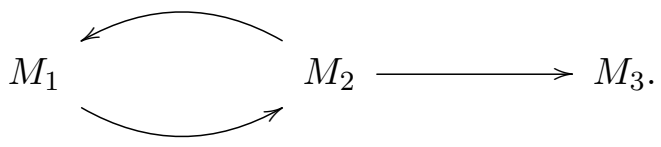

The total phase space of such a system would be the product $M=M_{1} \times M_{2} \times M_{3}$ and the dynamics would be governed by a vector field $X$ of the form

$$
X\left(x_{1}, x_{2}, x_{3}\right)=\left(X_{1}\left(x_{1}, x_{2}\right), X_{2}\left(x_{2}, x_{1}\right), X_{3}\left(x_{3}, x_{2}\right)\right) .
$$

How are we then to interpret the diagram of the form $M \bigcirc$ ? And why would we want to? Here is a two part answer. We interpret this diagram as a vector field $X$ on the manifold $M$ of the form

$$
X(x)=w(x, x),
$$

where $w: M \times M \rightarrow T M$ is an open system with $w\left(x_{1}, x_{2}\right) \in T_{x_{1}} M$ for all $\left(x_{1}, x_{2}\right) \in M \times M$. This seems a bit strange and pedantic, but it is useful. Consider a vector field $Z$ on $M \times M \times M$ of the form

$$
Z\left(x_{1}, x_{2}, x_{3}\right)=\left(w\left(x_{1}, x_{2}\right), w\left(x_{2}, x_{1}\right), w\left(x_{3}, x_{2}\right)\right),
$$

where $w$ is the open system above. The vector field $Z$ on $M$ models the dynamics of a system consisting of three interacting subsystems with the first driving the second, the second driving the first and the third just as in (1.1), only now all the subsystems have isomorphic phase spaces. It is not hard to check that the diagonal

$$
\Delta_{M}:=\left\{\left(x_{1}, x_{2}, x_{3}\right) \in M \times M \times M \mid x_{1}=x_{2}=x_{3}\right\}
$$

is an invariant submanifold for the vector field $Z$. According to the philosophy we brought up in the first paragraph of the paper the invariance of $\Delta_{M}$ should be seen as coming from a map of dynamical systems. And indeed the diagonal map

$$
\delta: M \rightarrow M \times M \times M, \quad \delta(x)=(x, x, x)
$$

gives rise to a map of dynamical systems $\delta:(M, X) \rightarrow(M \times M \times M, Z)$. The main result of the paper, Theorem 3.11, implies that this map of dynamical systems is induced by the map of graphs

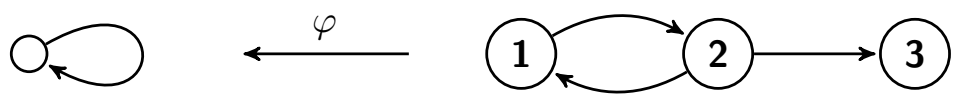

which is a graph fibration. We note that the vector field $Z$ has groupoid symmetry in the sense of Golubitsky et al. [7, 8] and [5]. For us, however, the groupoid invariance of $Z$ is, in some sense, 
incidental. It is a consequence of the fact that $Z$ is assembled out of the triple of open systems which lies in the image of the map $\varphi^{*}$ of Theorem 3.8 and that $\varphi$ happens to be surjective.

The paper is organized as follows. We start by defining the category DS of continuous time dynamical systems. We recall the definition of a directed multigraph, define the notion of a network of manifolds and the total space of the network. We recall the notion of an open system and discuss interconnections of open systems. We show how a network of manifolds naturally leads to a collection of spaces of open systems that can be interconnected. We then prove our main result, Theorem 3.11: fibrations of networks of manifolds give rise to maps of dynamical systems. We end the paper with a collection of examples.

\section{Definitions and constructions}

We start by defining what we mean by a continuous time dynamical system and by a map between two such systems.

Definition 2.1. A continuous time dynamical system is a vector field on a manifold. More formally it is a pair $(M, X)$, where $X$ is a vector field on a manifold $M$.

A map from a dynamical system $(M, X)$ to a dynamical system $(N, Y)$ is a smooth map $f: M \rightarrow N$ that intertwines the two vector fields:

$$
D f \circ X=Y \circ f
$$

where $D f: T M \rightarrow T N$ denotes the differential of $f$. One also says that the vector fields $X$ and $Y$ are $f$-related.

Notation 2.2 (the category DS of dynamical systems). Continuous time dynamical systems and maps of dynamical systems form a category. We denote it by DS.

\subsection{Graphs and manifolds}

Throughout the paper graphs are finite directed multigraphs, possibly with loops. More precisely, we use the following definition:

Definition 2.3. A graph $G$ consists of two finite sets $G_{1}$ (of arrows, or edges), $G_{0}$ (of nodes, or vertices) and two maps $\mathfrak{s}, \mathfrak{t}: G_{1} \rightarrow G_{0}$ (source, target); we write

$$
G=\left\{G_{1} \rightrightarrows G_{0}\right\} .
$$

The set $G_{1}$ may be empty, i.e., we may have $G=\left\{\varnothing \rightrightarrows G_{0}\right\}$, making $G$ a disjoint collection of vertices with no arrows between them.

Definition 2.4. A map of graphs $\varphi: A \rightarrow B$ from a graph $A$ to a graph $B$ is a pair of maps $\varphi_{1}: A_{1} \rightarrow B_{1}, \varphi_{0}: A_{0} \rightarrow B_{0}$ taking edges of $A$ to edges of $B$, nodes of $A$ to nodes of $B$ so that for any edge $\gamma$ of $A$ we have

$$
\varphi_{0}(\mathfrak{s}(\gamma))=\mathfrak{s}\left(\varphi_{1}(\gamma)\right) \quad \text { and } \quad \varphi_{0}(\mathfrak{t}(\gamma))=\mathfrak{t}\left(\varphi_{1}(\gamma)\right)
$$

We often omit the indices 0 and 1 and write $\varphi(\gamma)$ for $\varphi_{1}(\gamma)$ and $\varphi(a)$ for $\varphi_{0}(a)$.

Remark 2.5. The collection of finite (directed multi-)graphs and maps of graphs form a category Graph.

In order to construct networks from graphs we need to have a consistent way of assigning manifolds to nodes of our graphs. We formalize this idea by making the collection of graphs with manifolds assigned to vertices into a category Graph/Man. 
Definition 2.6 (category of networks of manifolds Graph/Man). A network of manifolds is a pair $(G, \mathcal{P})$, where $G$ is a (finite directed multi-)graph and $\mathcal{P}: G_{0} \rightarrow$ Man is a function that assigns to each node $a$ of $G$ a manifold $\mathcal{P}(a)$. We think of $\mathcal{P}$ as an assignment of phase spaces to the nodes of the graph $G$, and for this reason we refer to $\mathcal{P}$ as a phase space function.

Networks of manifolds form a category Graph/Man. Its objects are are pairs $(G, \mathcal{P})$ as above. A morphism $\varphi$ from $(G, \mathcal{P})$ to $\left(G^{\prime}, \mathcal{P}^{\prime}\right)$ is a map of graphs $\varphi: G \rightarrow G^{\prime}$ with

$$
\mathcal{P}^{\prime} \circ \varphi=\mathcal{P} \text {. }
$$

Notation 2.7. Given a category $\mathscr{C}$ we denote the opposite category by $\mathscr{C}^{\text {op }}$, i.e. the category with all of the same objects and all of the arrows reversed. We adhere to the convention that a contravariant functor from a category $\mathscr{C}$ to a category $\mathscr{D}$ is a covariant functor

$$
F: \mathscr{C}^{\mathrm{op}} \rightarrow \mathscr{D} .
$$

Then for any morphism $c \stackrel{\gamma}{\rightarrow} c^{\prime}$ of $\mathscr{C}$ we have $F(c) \stackrel{F(\gamma)}{\longleftarrow} F\left(c^{\prime}\right)$ in $\mathscr{D}$.

Next we recall the notion of a product in a category $\mathscr{C}$. We will use them in two instances: when $\mathscr{C}$ is the category Man of smooth finite dimensional manifolds and smooth maps and when $\mathscr{C}$ is the category Vect of real (but not necessarily finite dimensional) vector spaces and linear maps.

Definition 2.8. A product of a family $\left\{c_{s}\right\}_{s \in S}$ of objects in a category $\mathscr{C}$ indexed by a set $S$ is an object $\prod_{s^{\prime} \in S} c_{s^{\prime}}$ of $\mathscr{C}$ together with a family of morphisms $\left\{\pi_{s}: \prod_{s^{\prime} \in S} c_{s^{\prime}} \rightarrow c_{s}\right\}_{s \in S}$ with the following universal property: given an object $c^{\prime}$ of $\mathscr{C}$ and a family of morphisms $\left\{f_{s}: c^{\prime} \rightarrow c_{s}\right\}_{s \in S}$ there is a unique morphism $f: c^{\prime} \rightarrow \prod_{s \in S} c_{s}$ with

$$
\pi_{s} \circ f=f_{s} \quad \text { for all } s \in S .
$$

Remark 2.9. If a product exists then it is unique up to a unique isomorphism [1].

Lemma 2.10. The category of manifolds Man has (finite) categorical products.

Proof. There are several ways to construct categorical products in Man. The first one uses Cartesian products: given a family $\left\{M_{s}\right\}_{s \in S}$ of manifolds indexed by an $n$-element set $S$, order the elements of $S: S=\left\{s_{1}, \ldots, s_{n}\right\}$. Set

$$
\prod_{s \in S} M_{s}=\prod_{i=1}^{n} M_{s_{i}}
$$

where the right hand side is the Cartesian product. The projections $p_{s_{j}}: \prod_{i=1}^{n} M_{s_{i}} \rightarrow M_{s_{j}}$ are just projections on the $j$-th factor. It is easy to check that a product constructed this way has the requisite universal property. In particular, if we choose two different orderings of elements of $S$, the resulting products are canonically isomorphic. This construction is convenient for writing down examples.

However, for proving the results below, such as Proposition 2.15, it is better to have a construction of the product that does not involve a choice of ordering of the indexing set in question. This may be done as follows. Given a family $\left\{M_{s}\right\}_{s \in S}$ of manifolds, denote by $\bigsqcup_{s \in S} M_{s}$ their disjoint union ${ }^{1}$. Now define

$$
\prod_{s \in S} M_{s}:=\left\{x: S \rightarrow \bigsqcup_{s \in S} M_{s} \mid x(s) \in M_{s} \text { for all } s \in S\right\} .
$$

\footnotetext{
${ }^{1}$ It may be defined by $\bigsqcup_{s \in S} M_{s}=\bigcup_{s \in S} M_{s} \times\{s\}$.
} 
The projection maps $\pi_{s}: \prod_{s^{\prime} \in S} M_{s^{\prime}} \rightarrow M_{s}$ are defined by

$$
\pi_{s}(x)=x(s)
$$

We denote $x(s) \in M_{s}$ by $x_{s}$ and think of it as the $s^{\text {th }}$ "coordinate" of an element $x \in \prod_{s \in S} M_{s}$. Equivalently we may think of elements of the categorical product $\prod_{s \in S} M_{s}$ as unordered tuples $\left(x_{s}\right)_{s \in S}$ with $x_{s} \in M_{s}$.

Lemma 2.11. The category of vector spaces Vect has finite categorical products.

Sketch of proof. Just as in the proof of Lemma 2.10 the finite products in Vect can be constructed as vector spaces of ordered tuples of vectors, that is, as Cartesian products. Categorical products in Vect can also be constructed as unordered tuples of vectors.

Definition 2.12 (total phase space of a network $(G, \mathcal{P})$ ). For a pair $(G, \mathcal{P})$ consisting of a finite graph $G$ and an assignment $\mathcal{P}: G_{0} \rightarrow$ Man, that is, for an object $(G, \mathcal{P})$ of Graph/Man we set

$$
\mathbb{P} G \equiv \mathbb{P}(G, \mathcal{P}):=\prod_{a \in G_{0}} \mathcal{P}(a)
$$

the categorical product of manifolds attached to the nodes of the graph $G$ by the phase space function $\mathcal{P}$ and call the resulting manifold $\mathbb{P} G$ the total phase space of the network $(G, \mathcal{P})$.

Example 2.13. Consider the graph

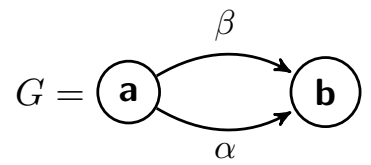

Define $\mathcal{P}: G_{0} \rightarrow$ Man by $\mathcal{P}(a)=S^{2}$ (the two sphere) and $\mathcal{P}(b)=S^{3}$. Then

$$
\mathbb{P}(G, \mathcal{P})=S^{2} \times S^{3} .
$$

Notation 2.14. If $G=\{\varnothing \rightrightarrows\{a\}\}$ is a graph with one node $a$ and no arrows, we write $G=\{a\}$. Then for any phase space function $\mathcal{P}: G_{0}=\{a\} \rightarrow$ Man we abbreviate $\mathbb{P}(\{\varnothing \rightrightarrows\{a\}\}, \mathcal{P}:\{a\} \rightarrow$ Man) as $\mathbb{P} a$.

Proposition 2.15. The assignment

$$
(G, \mathcal{P}) \mapsto \mathbb{P} G:=\prod_{a \in G_{0}} \mathcal{P}(a)
$$

of phase spaces to networks extends to a contravariant functor

$$
\mathbb{P}:(\text { Graph/Man })^{\text {op }} \rightarrow \text { Man. }
$$

Proof. Suppose $\varphi:(G, \mathcal{P}) \rightarrow\left(G^{\prime}, \mathcal{P}^{\prime}\right)$ is a morphism in Graph/Man. That is, suppose $\varphi: G \rightarrow$ $G^{\prime}$ is a map of graphs with $\mathcal{P}^{\prime} \circ \varphi=\mathcal{P}$. We need to define a map of manifolds

$$
\mathbb{P} \varphi: \mathbb{P} G^{\prime} \rightarrow \mathbb{P} G \text {. }
$$

Since by definition $\mathbb{P} G$ is the product $\prod_{a \in G_{0}} \mathcal{P}(a)$, the universal property of products implies that in order to define $\mathbb{P} \varphi$ it is enough to define a family of maps

$$
\left\{(\mathbb{P} \varphi)_{a}: \mathbb{P} G^{\prime} \rightarrow \mathcal{P}(a)\right\}_{a \in G_{0}} .
$$


For any node $a^{\prime}$ of $G^{\prime}$ we have the canonical projection

$$
\pi_{a^{\prime}}^{\prime}: \mathbb{P} G^{\prime} \rightarrow \mathcal{P}^{\prime}\left(a^{\prime}\right)
$$

We therefore define

$$
(\mathbb{P} \varphi)_{a}:=\pi_{\varphi_{a}}^{\prime}: \mathbb{P} G^{\prime} \rightarrow \mathcal{P}^{\prime}(\varphi(a))=\mathcal{P}(a)
$$

for all $a \in G_{0}$. By the universal property of the product $\mathbb{P} G=\prod_{a \in G_{0}} \mathcal{P}(a)$ this defines the desired $\operatorname{map} \mathbb{P} \varphi: \mathbb{P} G^{\prime} \rightarrow \mathbb{P} G$.

The universal property of products also implies that the map $\mathbb{P}$ on morphisms of Graph/Man as defined above is actually a functor. That is,

$$
\mathbb{P}(\psi \circ \varphi)=\mathbb{P} \varphi \circ \mathbb{P} \psi
$$

for any pair $(\psi, \varphi)$ of composable morphisms in Graph/Man.

Remark 2.16. Proposition 2.15 is an instance of a category-theoretic result that holds in greater generality. Namely, given a category $\mathscr{C}$ with finite products consider the category FinSet/ $\mathscr{C}$ whose objects are pairs $(X, P)$, where $X$ is a finite set and $P$ is a function that assignes to each element of $X$ an object of $\mathscr{C}$. The morphisms are commuting triangles. There is a contravariant functor $\mathbb{P}:(\text { FinSet } / \mathscr{C})^{\mathrm{op}} \rightarrow \mathscr{C}$ which on objects is given by

$$
\mathbb{P}(X, P)=\prod_{x \in X} P(x) .
$$

Example 2.17. Suppose $G$ is a graph with two nodes $a, b$ and no edges, $G^{\prime}$ is a graph with one node $\{c\}$ and no edges, $\mathcal{P}^{\prime}(c)$ is a manifold $M$, and $\varphi: G \rightarrow G^{\prime}$ is the only possible map of graphs (it sends both nodes to $c$ ). Suppose further that $\mathcal{P}: G_{0} \rightarrow$ Man is given by $\mathcal{P}(a)=M=\mathcal{P}(b)$ (so that $\mathcal{P}^{\prime} \circ \varphi=\mathcal{P}$ ). Then $\mathbb{P} G^{\prime} \simeq M$,

$$
\mathbb{P} G=\left\{\left(x_{a}, x_{b}\right) \mid x_{a} \in \mathcal{P}(a), x_{b} \in \mathcal{P}(b)\right\} \simeq M \times M,
$$

and $\mathbb{P} \varphi: M \rightarrow M \times M$ is the unique map with $(\mathbb{P} \varphi(x))_{a}=x$ and $(\mathbb{P} \varphi(x))_{b}=x$ for all $x \in \mathbb{P} G^{\prime}$. Thus $\mathbb{P} \varphi: M \rightarrow M \times M$ is the diagonal map $x \mapsto(x, x)$.

Example 2.18. Let $(G, \mathcal{P}),\left(G^{\prime}, \mathcal{P}^{\prime}\right)$ be as in Example 2.17 above and $\psi:\left(G^{\prime}, \mathcal{P}^{\prime}\right) \rightarrow(G, \mathcal{P})$ be the map that sends the node $c$ to $a$. Then $\mathbb{P} \psi: \mathbb{P} G \rightarrow \mathbb{P} G^{\prime}$ is the map that sends $\left(x_{a}, x_{b}\right)$ to $x_{a}$.

Remark 2.19. If $(G, \mathcal{P})$ is a graph with a phase function, that is, an object of Graph/Man, and $\varphi: H \rightarrow G$ a map of graphs then $\mathcal{P} \circ \varphi: H \rightarrow$ Man is a phase function and $\varphi:(H, \mathcal{P} \circ \varphi) \rightarrow(G, \mathcal{P})$ is a morphism in Graph/Man. We then have a map of manifolds

$$
\mathbb{P} \varphi: \mathbb{P}(H, \mathcal{P} \circ \varphi) \rightarrow \mathbb{P}(G, \mathcal{P}) .
$$

Similarly, a commutative diagram

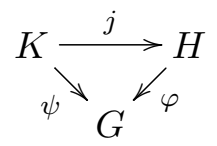

of maps of graphs and a phase space function $\mathcal{P}: G \rightarrow$ Man give rise to the commutative diagram of maps of manifolds

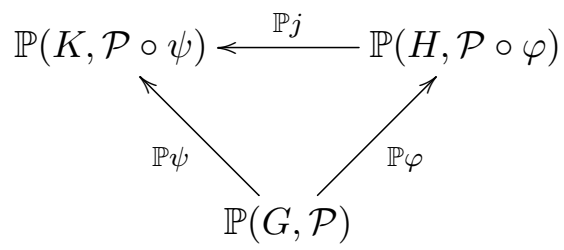




\subsection{Embeddings and submersions from maps of graphs}

As we said in the introduction, the main goal of this paper is to construct maps of dynamical systems from graph fibrations. In Proposition 2.15 we showed that a map of networks $\varphi:(G, \mathcal{P}) \rightarrow\left(G^{\prime}, \mathcal{P}^{\prime}\right)$ defines a map of manifolds $\mathbb{P} \varphi: \mathbb{P}\left(G^{\prime}, \mathcal{P}^{\prime}\right) \rightarrow \mathbb{P}(G, \mathcal{P})$. In this subsection we prove that:

1) If the map of graphs $\varphi: G \rightarrow G^{\prime}$ is injective on nodes, then $\mathbb{P} \varphi$ is a surjective submersion,

2) if the map of graphs $\varphi: G \rightarrow G^{\prime}$ is surjective on nodes, then $\mathbb{P} \varphi$ is an embedding.

(Recall that a smooth map between two manifolds is a submersion if its differential is onto at every point. A smooth map between two manifolds is an embedding if it is 1-1, its differential is 1-1 everywhere and it is a homeomorphism onto its image.) Combined with Theorem 3.11 below, this shows that surjective fibrations of networks of manifolds give rise to invariant dynamical subsystems and injective fibrations give rise to projections of dynamical systems.

Lemma 2.20. Suppose $\varphi:(G, \mathcal{P}) \rightarrow\left(G^{\prime}, \mathcal{P}^{\prime}\right)$ is a map of networks of manifolds such that the map on nodes, $\varphi_{0}: G_{0} \rightarrow G_{0}^{\prime}$, is surjective. Then $\mathbb{P} \varphi: \mathbb{P} G^{\prime} \rightarrow \mathbb{P} G$ is an embedding whose image is the "polydiagonal"

$$
\Delta_{\varphi}=\left\{x \in \mathbb{P} G \mid x_{a}=x_{b} \text { whenever } \varphi(a)=\varphi(b)\right\} .
$$

Proof. Assume first for simplicity that $G^{\prime}$ has only one vertex $*$ and $\mathcal{P}^{\prime}(*)=M$. Then for any vertex $a$ of $G$ we have

$$
\mathcal{P}(a)=\mathcal{P}^{\prime}(\varphi(a))=\mathcal{P}^{\prime}(*)=M,
$$

$\mathbb{P} G^{\prime}=M$ and $\mathbb{P} G=M \times \cdots \times M\left(\left|G_{0}\right|\right.$ copies $)$, where as before $G_{0}$ is the set of vertices of the graph $G$. In this case the proof of Proposition 2.15 shows that the map $\mathbb{P} \varphi: M \rightarrow M^{G_{0}}$ is of the form

$$
\mathbb{P} \varphi(x)=(x, \ldots, x)
$$

for all $x \in M$. This is clearly an embedding. In general,

$$
\mathbb{P} \varphi: \mathbb{P} G^{\prime}=\prod_{a^{\prime} \in G_{0}^{\prime}} \mathcal{P}^{\prime}\left(a^{\prime}\right) \rightarrow \prod_{a^{\prime} \in G_{0}^{\prime}}\left(\prod_{a \in \varphi^{-1}\left(a^{\prime}\right)} \mathcal{P}(a)\right)=\mathbb{P} G
$$

is the product of maps of the form

$$
\mathcal{P}^{\prime}\left(a^{\prime}\right) \rightarrow \prod_{a \in \varphi^{-1}\left(a^{\prime}\right)} \mathcal{P}(a), \quad x \mapsto(x, \ldots, x) .
$$

Lemma 2.21. Suppose $\varphi:(G, \mathcal{P}) \rightarrow\left(G^{\prime}, \mathcal{P}^{\prime}\right)$ is a map of networks of manifolds such that the map $\varphi_{0}: G_{0} \rightarrow G_{0}^{\prime}$ on nodes is injective. Then $\mathbb{P} \varphi: \mathbb{P} G^{\prime} \rightarrow \mathbb{P} G$ is a surjective submersion.

Proof. Since $\varphi: G \rightarrow G^{\prime}$ is injective, the set of nodes $G_{0}^{\prime}$ of $G^{\prime}$ can be partitioned as the disjoint union of the image $\varphi\left(G_{0}\right)$, which is a copy of $G_{0}$, and the complement. Hence

$$
\mathbb{P} G^{\prime} \simeq \prod_{a \in G_{0}} \mathcal{P}(\varphi(a)) \times \prod_{a^{\prime} \notin \varphi\left(G_{0}\right)} \mathcal{P}^{\prime}\left(a^{\prime}\right) \simeq \mathbb{P} G \times \prod_{a^{\prime} \notin \varphi\left(G_{0}\right)} \mathcal{P}^{\prime}\left(a^{\prime}\right)
$$

With respect to this identification of $\mathbb{P} G^{\prime}$ with $\mathbb{P} G \times \prod_{a^{\prime} \notin \varphi\left(G_{0}\right)} \mathcal{P}^{\prime}\left(a^{\prime}\right)$ the map $\mathbb{P} \varphi: \mathbb{P} G^{\prime} \rightarrow \mathbb{P} G$ is the projection

$$
\mathbb{P} G \times \prod_{a^{\prime} \notin \varphi\left(G_{0}\right)} \mathcal{P}^{\prime}\left(a^{\prime}\right) \rightarrow \mathbb{P} G .
$$

which is a surjective submersion. 


\subsection{Open systems and their interconnections}

Having set up a consistent way of assigning phase spaces to graphs, we now take up continuous time dynamical systems. We start by recalling a definition of an open (control) systems, which is essentially due to Brockett [3]. It is not the most general definition; it is more than enough for this paper.

Definition 2.22. A continuous time control system (or an open system) on a manifold $M$ is a surjective submersion $p: Q \rightarrow M$ from some manifold $Q$ together with a smooth map $F: Q \rightarrow T M$ so that

$$
F(q) \in T_{p(q)} M
$$

for all $q \in Q$. That is, the diagram

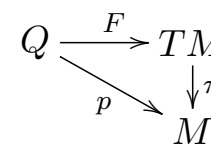
commutes. Here $\pi: T M \rightarrow M$ is the canonical projection.

Definition 2.23 (Control $(M \times U \rightarrow M))$. Given a manifold $M$ of "state variables" and a manifold $U$ of "control variables" we may consider control systems of the form

$$
F: M \times U \rightarrow T M, \quad F(x, u) \in T_{x} M \quad \text { for all } \quad(x, u) \in M \times U .
$$

The collection of all such control systems forms a vector space Control $(M \times U \rightarrow M)$. Explicitly

$$
\text { Control }(M \times U \rightarrow M):=\left\{F: M \times U \rightarrow T M \mid F(x, u) \in T_{x} M \text { for all }(x, u) \in M \times U\right\} .
$$

Now suppose we are given a finite family $\left\{F_{i}: M_{i} \times U_{i} \rightarrow T M_{i}\right\}_{i=1}^{N}$ of control systems and we want to somehow interconnect them to obtain a closed system $\mathscr{I}\left(F_{1}, \ldots, F_{N}\right)$, that is, a vector field on the product $\prod_{i} M_{i}$. What additional data do we need to define the interconnection map

$$
\mathscr{I}: \prod_{i} \operatorname{Control}\left(M_{i} \times U_{i} \rightarrow M_{i}\right) \rightarrow \Gamma\left(T\left(\prod_{i} M_{i}\right)\right) ?
$$

An answer is given by the following proposition:

Proposition 2.24. Given a family $\left\{p_{j}: M_{j} \times U_{j} \rightarrow M_{j}\right\}_{j=1}^{N}$ of projections on the first factor and a family of smooth maps $\left\{s_{j}: \prod M_{i} \rightarrow M_{j} \times U_{j}\right\}$ so that the diagrams

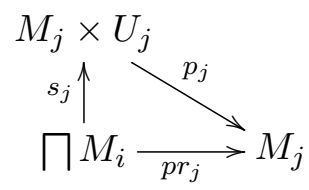

commute for each index $j$, there is an interconnection map $\mathscr{I}$ making the diagrams

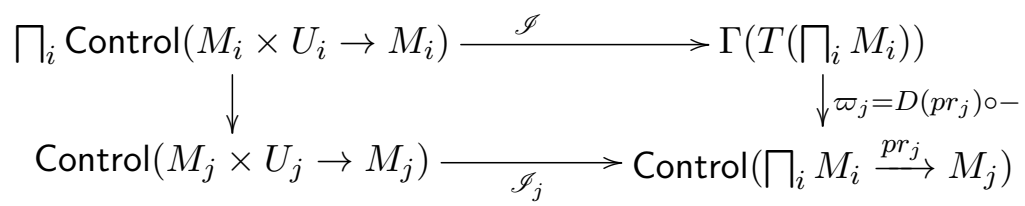

commute for each $j$. The components $\mathscr{I}_{j}$ of the interconnection map $\mathscr{I}$ are defined by $\mathscr{I}_{j}\left(F_{j}\right):=$ $F_{j} \circ s_{j}$ for all $j$, where $D\left(p r_{j}\right): T \sqcap M_{i} \rightarrow T M_{j}$ denotes the differential of the canonical projection $\mathrm{pr}_{j}: \prod M_{i} \rightarrow M_{j}$. 
Proof. The space of vector fields $\Gamma\left(T\left(\prod_{i} M_{i}\right)\right)$ on the product $\prod_{i} M_{i}$ is the product of vector spaces Control $\left(\prod_{i} M_{i} \rightarrow M_{j}\right)$ :

$$
\Gamma\left(T\left(\prod_{i} M_{i}\right)\right)=\prod_{j} \text { Control }\left(\prod_{i} M_{i} \stackrel{p r_{j}}{\longrightarrow} M_{j}\right) .
$$

In other words a vector field $X$ on the product $\prod_{i} M_{i}$ is a tuple $X=\left(X_{1}, \ldots, X_{N}\right)$, where

$$
X_{j}:=D\left(p r_{j}\right) \circ X .
$$

Each component $X_{j}: \prod_{i} M_{i} \rightarrow T M_{i}$ is a control system.

To define a map from a vector space into a product of vector spaces it is enough to define a map into each of the factors. We have canonical projections

$$
\pi_{j}: \prod_{i} \operatorname{Control}\left(M_{i} \times U_{i} \rightarrow M_{i}\right) \rightarrow \operatorname{Control}\left(M_{j} \times U_{j} \rightarrow M_{j}\right), \quad j=1, \ldots, N .
$$

Consequently to define the interconnection map $\mathscr{I}$ it is enough to define the maps

$$
\mathscr{I}_{j}: \operatorname{Control}\left(M_{j} \times U_{j} \rightarrow M_{j}\right) \rightarrow \operatorname{Control}\left(\prod_{i} M_{i} \stackrel{p r_{j}}{\longrightarrow} M_{j}\right) \text {. }
$$

for each index $j$. We therefore define the maps $\mathscr{I}_{j}: \operatorname{Control}\left(M_{j} \times U_{j} \rightarrow M_{j}\right) \rightarrow \operatorname{Control}\left(\prod_{i} M_{i} \stackrel{p r_{j}}{\longrightarrow}\right.$ $\left.M_{j}\right), 1 \leq j \leq N$, by

$$
\mathscr{I}_{j}\left(F_{j}\right):=F_{j} \circ s_{j} .
$$

Remark 2.25. It will be useful for us to remember that the canonical projections

$$
\varpi_{j}: \Gamma\left(T \prod M_{i}\right) \rightarrow \text { Control }\left(\prod M_{i} \rightarrow M_{j}\right)
$$

are given by

$$
\varpi_{j}(X)=D\left(p r_{j}\right) \circ X,
$$

where as before $D\left(p r_{j}\right): T \sqcap M_{i} \rightarrow T M_{j}$ are the differentials of the canonical projections $p r_{j}: \prod M_{i} \rightarrow M_{j}$.

\subsection{Interconnections and graphs}

We next explain how finite directed graphs whose nodes are decorated with phase spaces, that is, networks of manifolds in the sense of Definition 2.6 give rise to interconnection maps. To do this precisely it is useful to have a notion of input trees of a directed graph. This notion is a generalization of the notion of an input set of Golubitsky et al. (op. cit.) [7, 8]. Given a graph, an input tree $I(a)$ of a vertex $a$ is - roughly - the vertex itself and all of the arrows leading into it. We want to think of this as a graph in its own right, as follows.

Definition 2.26 (input tree). Given a vertex $a$ of a graph $G$ we define the input tree $I(a)$ to be a graph with the set of vertices $I(a)_{0}$ given by

$$
I(a)_{0}:=\{a\} \sqcup \mathfrak{t}^{-1}(a),
$$

where, as before, the set $\mathfrak{t}^{-1}(a)$ is the set of arrows in $G$ with target $a$. The set of edges $I(a)_{1}$ of the input tree is the set of pairs

$$
I(a)_{1}:=\left\{(a, \gamma) \mid \gamma \in G_{1}, \mathfrak{t}(\gamma)=a\right\},
$$


and the source and target maps $I(a)_{1} \rightrightarrows I(a)_{0}$ are defined by

$$
\mathfrak{s}(a, \gamma)=\gamma \quad \text { and } \quad \mathfrak{t}(a, \gamma)=a
$$

In pictures,

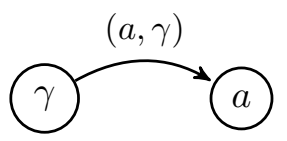

Example 2.27. Consider the graph

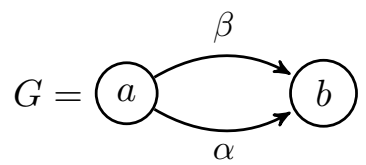

as in Example 2.13. Then the input tree $I(a)$ is the graph with one node $a$ and no edges: $I(a)=\{a\}$ (see Notation 2.14). The input tree $I(b)$ has three nodes and two edges:

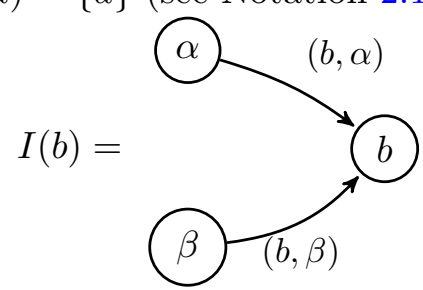

Notice that our definition of input tree "pulls apart" multiple edges coming from a common vertex.

Remark 2.28. For each node $a$ of a graph $G$ we have a natural map of graphs

$$
\xi=\xi_{a}: I(a) \rightarrow G
$$

It is defined by sending the edge of the form $\gamma \stackrel{(a, \gamma)}{\longrightarrow} a$ to the edge $\mathfrak{s}(\gamma) \stackrel{\gamma}{\rightarrow} a$. Note that the map $\xi$ need not be injective on vertices.

Proposition 2.29. Given a graph $G$ with a phase space function $\mathcal{P}: G_{0} \rightarrow$ Man, that is, a network $(G, \mathcal{P})$ of manifolds, we have commutative diagrams of maps of manifolds

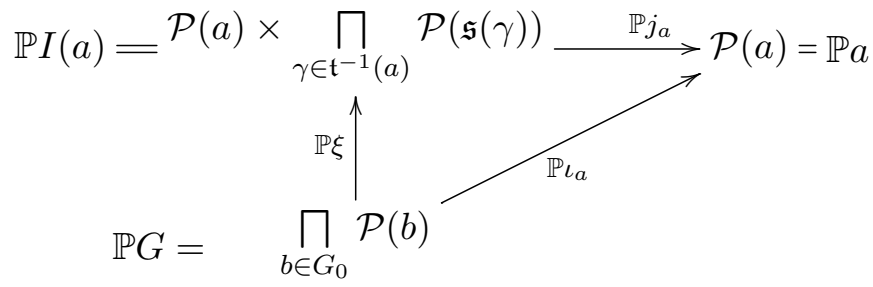

for each node a of the graph $G$.

Proof. Let $a$ be a node of the graph $G$. We then have a graph $\{a\}$ with one node and no arrows. Denote the inclusion of $\{a\}$ in $G$ by $\iota_{a}$ and the inclusion into its input tree $I(a)$ by $j_{a}$. Then the diagram of maps of graphs

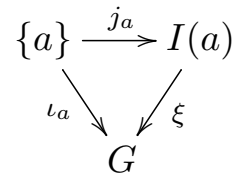


commutes. By Remark 2.19 we have a commuting diagram of maps of manifolds

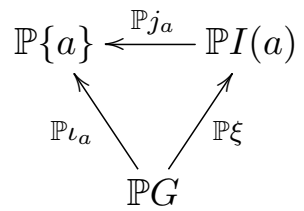

Let us now examine more closely the map $\mathbb{P} j_{a}: \mathbb{P} I(a) \rightarrow \mathbb{P} a$.

Since the set of nodes $I(a)_{0}$ of the input tree $I(a)$ is the disjoint union

$$
I(a)_{0}=\{a\} \sqcup \mathfrak{t}^{-1}(a),
$$

and since $\xi_{a}(\gamma)=\mathfrak{s}(\gamma)$ for any $\gamma \in \mathfrak{t}^{-1}(a) \subset I(a)_{0}$, we have

$$
\mathbb{P} I(a)=\mathcal{P}(a) \times \prod_{\gamma \in \mathfrak{t}^{-1}(a)} \mathcal{P}(\mathfrak{s}(\gamma)) .
$$

Since $j_{a}:\{a\} \rightarrow I(a)_{0}=\{a\} \sqcup \mathfrak{t}^{-1}(a)$ is the inclusion,

$$
\mathbb{P} j_{a}: \mathbb{P} I(a) \rightarrow \mathbb{P} a
$$

is the projection

$$
\mathcal{P}(a) \times \prod_{\gamma \in \mathfrak{t}^{-1}(a)} \mathcal{P}(\mathfrak{s}(\gamma)) \rightarrow \mathbb{P} a
$$

Similarly

$$
\mathbb{P} \iota_{a}: \mathbb{P} G \rightarrow \mathbb{P} a
$$

is the projection

$$
\prod_{b \in G_{0}} \mathcal{P}(b) \rightarrow \mathcal{P}(a)
$$

The proposition follows from these two observations.

Example 2.30. Suppose

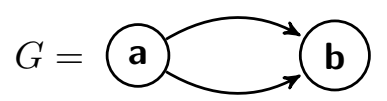

is a graph as in Example 2.13 and suppose $\mathcal{P}: G_{0} \rightarrow$ Man is a phase space function. Then

$$
\mathbb{P} I(b) \simeq \mathcal{P}(a) \times \mathcal{P}(a) \times \mathcal{P}(b),
$$

$\mathbb{P} j_{b}$ is the projection $\mathcal{P}(a) \times \mathcal{P}(a) \times \mathcal{P}(b) \rightarrow \mathcal{P}(b)$, and

$$
\operatorname{Control}(\mathbb{P} I(b) \rightarrow \mathbb{P} b)=\operatorname{Control}(\mathcal{P}(a) \times \mathcal{P}(a) \times \mathcal{P}(b) \rightarrow \mathcal{P}(b))
$$

On the other hand $\mathbb{P} I(a)=\mathcal{P}(a), \mathbb{P} j_{a}: \mathcal{P}(a) \rightarrow \mathcal{P}(a)$ is the identity map and

$$
\operatorname{Control}(\mathbb{P} I(a) \rightarrow \mathbb{P} a)=\Gamma(T \mathcal{P}(a)),
$$

the space of vector fields on the manifold $\mathcal{P}(a)$. 
Notation 2.31. Given a network $(G, \mathcal{P})$ of manifolds we have a product of vector spaces

$$
\operatorname{Ctr}(G, \mathcal{P}):=\prod_{a \in G_{0}} \operatorname{Control}(\mathbb{P} I(a) \rightarrow \mathbb{P} a)
$$

The elements of $\operatorname{Ctr}(G, \mathcal{P})$ are unordered tuples of $\left(w_{a}\right)_{a \in G_{0}}$ of control systems (q.v. Lemma 2.11). We may think of them as sections of the vector bundle $\bigsqcup_{a \in G_{0}} \operatorname{Control}(\mathbb{P} I(a) \rightarrow \mathbb{P} a) \rightarrow G_{0}$ over the vertices of $G$.

It is easy to see that Propositions 2.24 and 2.29 give us

Theorem 2.32. Given a network $(G, \mathcal{P})$ of manifolds, there exists a natural interconnection map

$$
\mathscr{I}: \prod_{a \in G_{0}} \operatorname{Control}(\mathbb{P} I(a) \rightarrow \mathbb{P} a) \rightarrow \Gamma(T \mathbb{P} G)
$$

with

$$
\varpi_{a} \circ \mathscr{I}\left(\left(w_{b}\right)_{b \in G_{0}}\right)=w_{a} \circ \mathbb{P} j_{a}
$$

for all nodes $a \in G_{0}$. Here $\varpi_{a}: \Gamma(T \mathbb{P} G) \rightarrow \operatorname{Control}\left(\mathbb{P} G_{0} \stackrel{\mathbb{P}_{\iota_{a}}}{\longrightarrow} \mathbb{P} a\right)$ are the projection maps; $\varpi_{a}=D\left(\mathbb{P} \iota_{a}\right)$ (q.v. Remark 2.25).

Example 2.33. Consider the graph $G$ as in Examples 2.13 and 2.30 with a phase space function $\mathcal{P}: G_{0} \rightarrow$ Man. Then the vector field

$$
X=\mathscr{I}\left(w_{a}, w_{b}\right): \mathcal{P}(a) \times \mathcal{P}(b) \rightarrow T \mathcal{P}(a) \times T \mathcal{P}(b)
$$

is of the form

$$
X(x, y)=\left(w_{a}(x), w_{b}(x, x, y)\right) \quad \text { for all } \quad(x, y) \in \mathcal{P}(a) \times \mathcal{P}(b)
$$

Example 2.34. Consider the graph

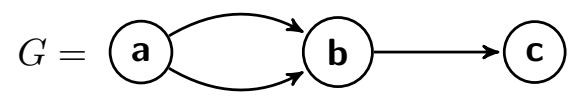

and let $\mathcal{P}: G_{0} \rightarrow$ Man be a phase space function. Then

$$
\left(\mathscr{I}\left(w_{a}, w_{b}, w_{c}\right)\right)(x, y, z)=\left(w_{a}(x), w_{b}(x, x, y), w_{c}(y, z)\right)
$$

for all $\left(w_{a}, w_{b}, w_{c}\right) \in \operatorname{Ctrl}(G, \mathcal{P})$ and all $(x, y, z) \in \mathcal{P}(a) \times \mathcal{P}(b) \times \mathcal{P}(c)$.

\section{Maps of dynamical systems from fibrations}

Following Boldi and Vigna [2] (see also [11]) we single out a class of maps of graphs called graph fibrations.

Definition 3.1. A map $\varphi: G \rightarrow G^{\prime}$ of directed graphs is a graph fibration if for any vertex $a$ of $G$ and any edge $e^{\prime}$ of $G^{\prime}$ ending at $\varphi(a)$ there is a unique edge $e$ of $G$ ending at $a$ with $\varphi(e)=e^{\prime}$. 
Example 3.2. The map of graphs

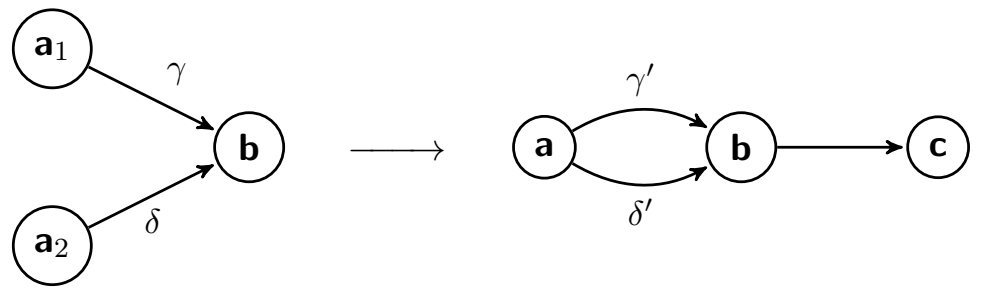

sending the edge $\gamma$ to $\gamma^{\prime}$ and the edge $\delta$ to $\delta^{\prime}$ is a graph fibration.

Remark 3.3. Given any map $\varphi: G \rightarrow G^{\prime}$ of graphs and a node $a$ of $G$ there is an induced map of input trees

$$
\varphi_{a}: \quad I(a) \rightarrow I(\varphi(a)) .
$$

On edges of $I(a)$ the map is defined by

$$
\varphi(a, \gamma):=(\varphi(a), \varphi(\gamma))
$$

(cf. Definition 2.26). Moreover the diagram of graphs

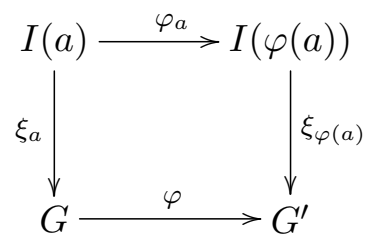

commutes (the map $\xi_{a}: I(a) \rightarrow G$ from an input tree to the original graph is defined in Remark 2.28).

Lemma 3.4. If $\varphi: G \rightarrow G^{\prime}$ is a graph fibration then the induced maps

$$
\varphi_{a}: I(a) \rightarrow I(\varphi(a))
$$

of input trees defined above are isomorphisms for all nodes a of $G$.

Proof. Given an edge $\left(\varphi(a), \gamma^{\prime}\right)$ of $I(\varphi(a))$ there is a unique edge $\gamma$ of $G$ with $\varphi(\gamma)=\gamma^{\prime}$ and $\mathfrak{t}(\gamma)=a$ and consequently $\varphi_{a}(a, \gamma)=\left(\varphi(a), \gamma^{\prime}\right)$. It follows that $\varphi_{a}$ is bijective on vertices and edges.

Remark 3.5. The converse is true as well: if the induced maps $\varphi_{a}: I(a) \rightarrow I(\varphi(a))$ are isomorphisms for all nodes $a$ of $G$ then $\varphi: G \rightarrow G^{\prime}$ is a graph fibration.

Recall that a map from a network $(G, \mathcal{P})$ to a network $\left(G^{\prime}, \mathcal{P}^{\prime}\right)$ is a map of graphs $\varphi: G \rightarrow G^{\prime}$ with the property that

$$
\mathcal{P}^{\prime} \circ \varphi=\mathcal{P} \text {. }
$$

Definition 3.6 (fibration of networks of manifolds). A map of networks $\varphi:(G, \mathcal{P}) \rightarrow\left(G^{\prime}, \mathcal{P}^{\prime}\right)$ of manifolds is a fibration if $\varphi: G \rightarrow G^{\prime}$ is a graph fibration.

Remark 3.7 (the category (Man/Graph) fib of networks of manifolds and fibrations). We note that the composit of two fibrations is again a fibration. Consequently networks of manifolds and fibrations form a category which we denote by (Man/Graph) $)_{\text {fib }}$. 
Theorem 3.8 below is our reason for singling out fibrations of networks.

Theorem 3.8. A fibration $\varphi:(G, \mathcal{P}) \rightarrow\left(G^{\prime}, \mathcal{P}^{\prime}\right)$ of networks induces a linear map

$$
\varphi^{*}: \operatorname{Ctrl}\left(G^{\prime}, \mathcal{P}^{\prime}\right) \rightarrow \operatorname{Ctrl}(G, \mathcal{P}) .
$$

Proof. Since

$$
\operatorname{Ctrl}(G, \mathcal{P})=\prod_{a \in G_{0}} \operatorname{Control}(\mathbb{P} I(a) \rightarrow \mathbb{P} a)
$$

is a product of vector spaces, the map $\varphi^{*}$ is uniquely determined by maps from $\operatorname{Ctrl}\left(G^{\prime}, \mathcal{P}^{\prime}\right)$ to the factors Control $(\mathbb{P} I(a) \rightarrow \mathbb{P} a), a \in G_{0}$. On the other hand we have canonical projections

$$
\pi_{b}: \operatorname{Ctrl}\left(G^{\prime}, \mathcal{P}^{\prime}\right)=\prod_{c \in G_{0}^{\prime}} \operatorname{Control}(\mathbb{P} I(c) \rightarrow \mathbb{P} c) \rightarrow \operatorname{Control}(\mathbb{P} I(b) \rightarrow \mathbb{P} b)
$$

for all $b \in G_{0}^{\prime}$. Hence in order to define the map $\varphi^{*}$ it is enough to define maps of vector spaces

$$
\varphi_{a}^{*}: \operatorname{Control}(\mathbb{P} I(\varphi(a)) \rightarrow \mathbb{P} \varphi(a)) \rightarrow \operatorname{Control}(\mathbb{P} I(a) \rightarrow \mathbb{P} a)
$$

for all nodes $a$ of the graph $G$. By Remark 3.3 the diagram

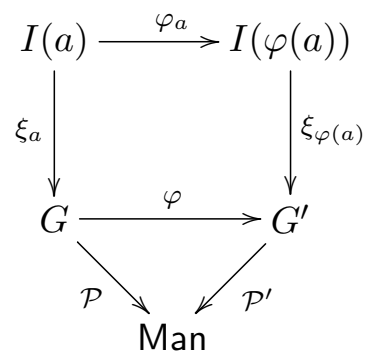

commutes for each $a \in G_{0}$. Let

$$
\left.\varphi\right|_{\{a\}}:\{a\} \rightarrow\{\varphi(a)\}
$$

denote the restriction of $\varphi: G \rightarrow G^{\prime}$ to the subgraph $\{a\} \hookrightarrow G$. It is easy to see that the diagrams

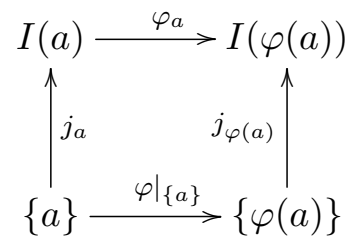

commutes as well. By Lemma 3.4 the map $\varphi_{a}$ is an isomorphism of graphs. Hence

$$
\mathbb{P} \varphi_{a}: \mathbb{P} I(a) \rightarrow \mathbb{P} I(\varphi(a))
$$

is an isomorphism of manifolds. Define

$$
\varphi_{a}^{*}: \operatorname{Control}(\mathbb{P} I(\varphi(a)) \rightarrow \mathbb{P} \varphi(a)) \rightarrow \operatorname{Control}(\mathbb{P} I(a) \rightarrow \mathbb{P} a)
$$

by

$$
\varphi_{a}^{*}(F)=D \mathbb{P}\left(\left.\varphi\right|_{\{a\}}\right) \circ F \circ\left(\mathbb{P} \varphi_{a}\right)^{-1}
$$


for all $F \in \operatorname{Control}(\mathbb{P} I(\varphi(a)))$. By the universal property of products this gives us the desired $\operatorname{map} \varphi^{*}$. Moreover the diagrams

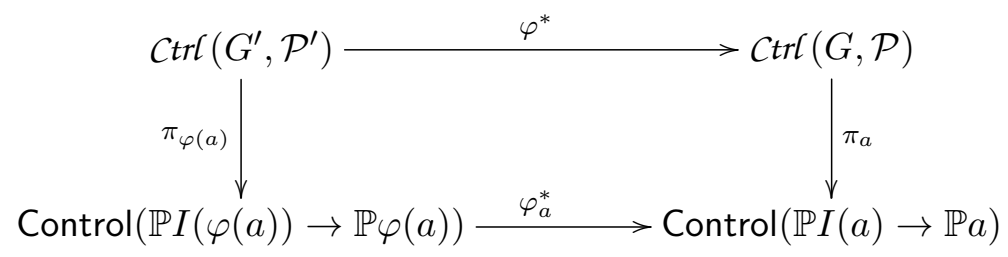

commute for all $a \in G_{0}$.

Example 3.9. We write down an example of the map $\varphi^{*}$ constructed in Theorem 3.8. Consider the graph fibration $\varphi: G \rightarrow G^{\prime}$ :

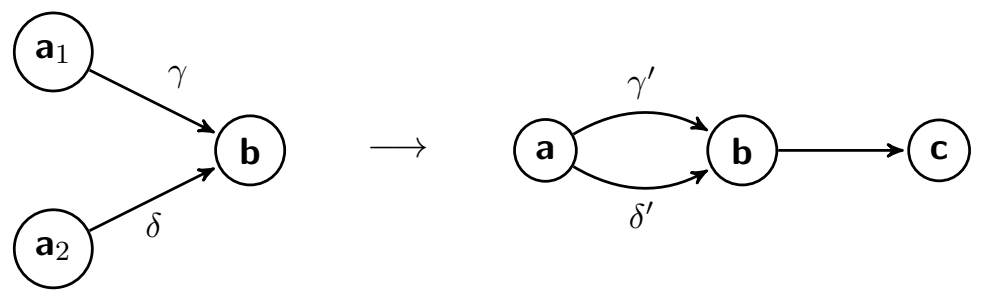

as in Example 3.2. Let $\mathcal{P}^{\prime}: G_{0}^{\prime} \rightarrow$ Man be a phase space function. Then

$$
\begin{gathered}
\operatorname{Ctrl}\left(G^{\prime}, \mathcal{P}^{\prime}\right)=\left\{\left(w_{a}: \mathcal{P}(a) \rightarrow T \mathcal{P}(a), w_{b}: \mathcal{P}^{\prime}(a) \times \mathcal{P}^{\prime}(a) \times \mathcal{P}^{\prime}(b) \rightarrow T \mathcal{P}(b),\right.\right. \\
\left.\left.w_{c}: \mathcal{P}(b) \times \mathcal{P}(c) \rightarrow T \mathcal{P}(c)\right)\right\} \\
\operatorname{Ctrl}\left(G, \mathcal{P}^{\prime} \circ \varphi\right)=\left\{\left(w_{a_{1}}^{\prime}: \mathcal{P}^{\prime}(a) \rightarrow \mathcal{P}^{\prime}(a), w_{a_{2}}: \mathcal{P}^{\prime}(a) \rightarrow \mathcal{P}^{\prime}(a)\right.\right. \\
\left.\left.w_{b}: \mathcal{P}^{\prime}(a) \times \mathcal{P}^{\prime}(a) \times \mathcal{P}^{\prime}(b) \rightarrow T \mathcal{P}^{\prime}(b)\right)\right\}
\end{gathered}
$$

and

$$
\varphi^{*}\left(w_{a}^{\prime}, w_{b}^{\prime}, w_{c}^{\prime}\right)=\left(w_{a}^{\prime}, w_{a}^{\prime}, w_{b}^{\prime}\right) .
$$

Remark 3.10 (the category DSN of dynamical systems on networks of manifolds). It is easy to see that if $\varphi:(G, \mathcal{P}) \rightarrow\left(G^{\prime}, \mathcal{P}^{\prime}\right)$ and $\psi:\left(G^{\prime}, \mathcal{P}^{\prime}\right) \rightarrow\left(G^{\prime \prime}, \mathcal{P}^{\prime \prime}\right)$ are two fibrations then

$$
(\psi \circ \varphi)^{*}=\varphi^{*} \circ \psi^{*}
$$

This can be interpreted as saying that the assignment

$$
(G, \mathcal{P}) \mapsto \operatorname{Ctr}(G, \mathcal{P})
$$

extends to a contravariant functor $\mathrm{Ctr}$ from the category (Man/Graph) fib $_{\text {of }}$ of networks of manifolds and fibrations to the category Vect of real vector spaces and linear maps. That is, on arrows,

$$
\operatorname{Ctrl} \varphi:=\varphi^{*}
$$

Grothendieck's construction (see for example [1]) applied to this functor produces a category DSN which we would like to call the category of (continuous time) dynamical systems on networks of manifolds. More explicitly the objects of the category DSN are triples

$$
\left(G, \mathcal{P}: G_{0} \rightarrow \text { Man, } w \in \operatorname{Ctrl}(G, \mathcal{P})\right),
$$

where as before $G$ is a finite directed graph, $\mathcal{P}$ is a phase space function and $w=\left(w_{a}\right)_{a \in G_{0}}$ is a tuple of control systems associated with the input trees of the graph $G$ and the function $\mathcal{P}$. 
A morphism from $\left(G^{\prime}, \mathcal{P}^{\prime}, w^{\prime}\right)$ to $(G, \mathcal{P}, w)$ is a graph fibration $\varphi: G \rightarrow G^{\prime}$ with $\mathcal{P}^{\prime} \circ \varphi=\mathcal{P}$ and $\varphi^{*} w^{\prime}=w$. Alternatively we may think of a map from $\left(G^{\prime}, \mathcal{P}^{\prime}, w^{\prime}\right)$ to $(G, \mathcal{P}, w)$ as a fibration of networks of manifolds $\varphi:(G, \mathcal{P}) \rightarrow\left(G^{\prime}, \mathcal{P}^{\prime}\right)$ with $\varphi^{*} w^{\prime}=w$.

Note that the Grothendieck construction also gives us a forgetful functor

$$
\mathrm{DSN} \rightarrow(\text { Man/Graph })_{\mathrm{fib}}{ }^{\text {op }}
$$

that simply forgets the open systems. On objects it is given by sending the triple $(G, \mathcal{P}, w)$ to the pair $(G, \mathcal{P})$.

Of course just because we can define a category and call it a category of dynamical systems on networks does not mean that this is a right thing to do. This said, Theorem 2.32 tells us that to every dynamical system on a network $(G, \mathcal{P}, w)$ we can assign a dynamical system $(\mathbb{P} G, \mathscr{I} w)$. We will next argue that this assignment actually extends to a functor

$$
\mathbb{P}: \mathrm{DSN} \rightarrow \mathrm{DS}
$$

from dynamical systems on networks to the category DS of dynamical systems (q.v. Definition 2.1 and Remark 2.2). The first step is to define the functor on arrows. We do it in Theorem 3.11 below which may be considered the main result of the paper.

Theorem 3.11. Let $\varphi:(G, \mathcal{P}) \rightarrow\left(G^{\prime}, \mathcal{P}^{\prime}\right)$ be a fibration of networks of manifolds. Then the pullback map

$$
\varphi^{*}: \operatorname{Ctrl}\left(G^{\prime}, \mathcal{P}^{\prime}\right) \rightarrow \operatorname{Ctrl}(G, \mathcal{P})
$$

constructed in Theorem 3.8 is compatible with the interconnection maps

$$
\mathscr{I}^{\prime}: \operatorname{Ctrl}\left(G^{\prime}, \mathcal{P}^{\prime}\right) \rightarrow \Gamma\left(T \mathbb{P} G^{\prime}\right) \quad \text { and } \quad \mathscr{I}: \operatorname{Ctrl}(G, \mathcal{P}) \rightarrow \Gamma(T \mathbb{P} G) .
$$

Namely for any collection $w^{\prime} \in \mathcal{C} \operatorname{tr}\left[\left(G^{\prime}, \mathcal{P}^{\prime}\right)\right.$ of open systems on the network $\left(G^{\prime}, \mathcal{P}^{\prime}\right)$ the diagram

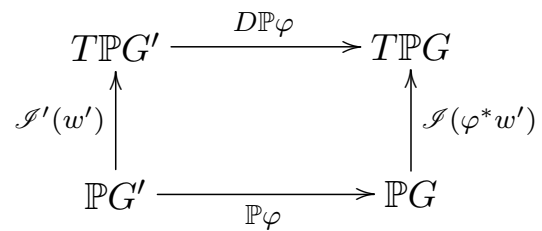

commutes. Consequently

$$
\mathbb{P} \varphi: \quad\left(\mathbb{P}\left(G^{\prime}, \mathcal{P}^{\prime}\right), \mathscr{I}^{\prime}\left(w^{\prime}\right)\right) \rightarrow\left(\mathbb{P}(G, \mathcal{P}), \mathscr{I}\left(\varphi^{*} w^{\prime}\right)\right)
$$

is a map of dynamical systems.

Proof. Recall that the manifold $\mathbb{P} G$ is the product $\prod_{a \in G_{0}} \mathbb{P} a$. Hence the tangent bundle bundle $T \mathbb{P} G$ is the product $\prod_{a \in G_{0}} T \mathbb{P} a$. The canonical projections

$$
T \mathbb{P} G \rightarrow T \mathbb{P} a
$$

are the differentials of the maps $\mathbb{P} \iota_{a}: \mathbb{P} G \rightarrow \mathbb{P} a$, where, as before, $\iota_{a}:\{a\} \hookrightarrow G$ is the canonical inclusion of graphs. Hence by the universal property of products, two maps into $T \mathbb{P} G$ are equal if and only if all their components are equal. Therefore, in order to prove that (3.1) commutes it is enough to show that

$$
D \mathbb{P} \iota_{a} \circ \mathscr{I}\left(\varphi^{*} w^{\prime}\right) \circ \mathbb{P} \varphi=D \mathbb{P} \iota_{a} \circ D \mathbb{P} \varphi \circ \mathscr{I}^{\prime}\left(w^{\prime}\right)
$$


for all nodes $a \in G_{0}$. By definition of the restriction $\left.\varphi\right|_{\{a\}}$ of $\varphi: G \rightarrow G^{\prime}$ to $\{a\} \hookrightarrow G$, the diagram

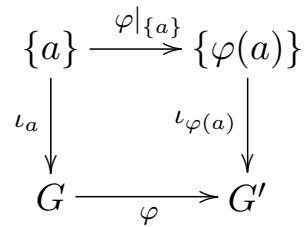

commutes. By the definition of the pullback map $\varphi^{*}$ and the interconnection maps $\mathscr{I}, \mathscr{I}^{\prime}$ the diagram

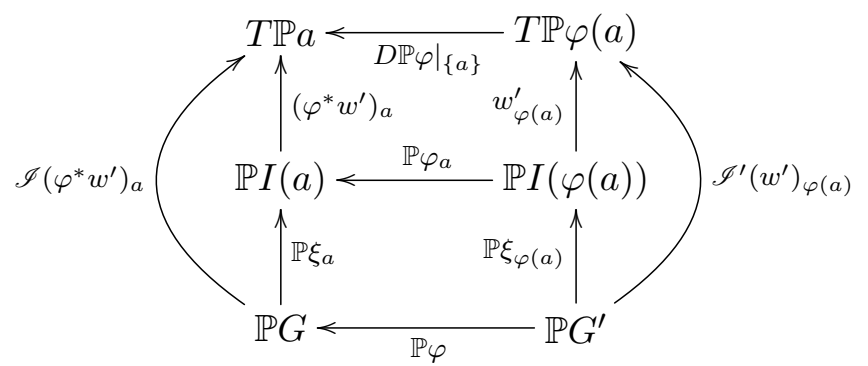

commutes as well. We now compute:

$$
\begin{aligned}
D \mathbb{P} \iota_{a} & \circ \mathscr{I}\left(\varphi^{*} w^{\prime}\right) \circ \mathbb{P} \varphi=\left(\mathscr{I}\left(\varphi^{*} w^{\prime}\right)\right)_{a} \circ \mathbb{P} \varphi \\
& =D \mathbb{P}\left(\left.\varphi\right|_{\{a\}}\right) \circ \mathscr{I}^{\prime}\left(w^{\prime}\right)_{\varphi(a)} \\
& =D \mathbb{P}\left(\left.\varphi\right|_{\{a\}}\right) \circ D \mathbb{P} \iota_{\varphi(a)} \circ \mathscr{I}^{\prime}\left(w^{\prime}\right) \\
& =D \mathbb{P}\left(\left.\iota_{\varphi(a)} \circ \varphi\right|_{\{a\}}\right) \circ \mathscr{I}^{\prime}\left(w^{\prime}\right) \\
& =D \mathbb{P}\left(\varphi \circ \iota_{a}\right) \circ \mathscr{I}^{\prime}\left(w^{\prime}\right) \\
& =D \mathbb{P}\left(\iota_{a}\right) \circ D \mathbb{P} \varphi \circ \mathscr{I}^{\prime}\left(w^{\prime}\right) .
\end{aligned}
$$

by definition of $\mathscr{I}^{\prime}\left(w^{\prime}\right)_{\varphi(a)}$

since $\mathbb{P}$ is a contravariant functor

by $(3.2)$

And we are done.

Corollary 3.12. The map

$$
\begin{aligned}
& \mathrm{DSN} \rightarrow \mathrm{DS} \\
& \left(\left(G^{\prime}, \mathcal{P}^{\prime}, w^{\prime}\right) \stackrel{\varphi}{\rightarrow}(G, \mathcal{P}, w)\right) \mapsto\left(\left(\mathbb{P} G^{\prime}, \mathscr{I}\left(w^{\prime}\right)\right) \stackrel{\mathbb{P} \varphi}{\longrightarrow}(\mathbb{P} G, \mathscr{I}(w))\right)
\end{aligned}
$$

is a functor.

Remark 3.13. Given a dynamical system on a network $(G, \mathcal{P}, w)$ we can forget the dynamics. This defines a functor

$$
\mathrm{DSN} \rightarrow(\text { Man/Graph })_{\text {fib }}
$$

from the category of dynamical systems on networks to a subcategory of the category of networks of manifolds whose maps are fibrations of networks (hence the subscript ${ }_{\text {fib }}$ ). Composing the functor above with the functor Man/Graph $\rightarrow G$ raph $^{\text {op }}$ forgets all the information except for the graph. This gives rise to a functor

$$
\mathrm{DSN} \rightarrow \mathrm{Graph}_{\mathrm{fib}}{ }^{\text {op }} \text {. }
$$

Here the superscript op indicates that the functor reverses the direction of arrows and the subscript ${ }_{\text {fib }}$ reminds us that the morphisms in the target category are the (opposite of the) graph fibrations.

These two functors from DSN to DS and to $\mathrm{Graph}_{\text {fib }}{ }^{\text {op }}$, respectively, allow us to interpret continuous time dynamical systems on networks both as dynamical systems and as graphs. 
We end the paper with examples.

Example 3.14. Consider the graph fibration

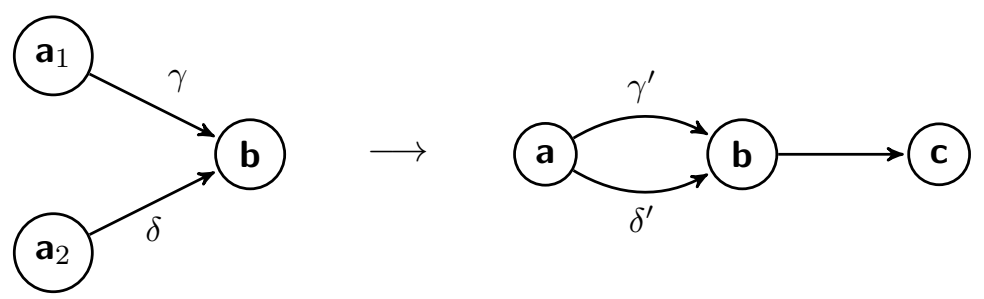

as in Examples 3.2 and 3.9. Let $\mathcal{P}^{\prime}: G_{0}^{\prime} \rightarrow$ Man be a phase space function and let $\mathcal{P}=\mathcal{P}^{\prime} \circ \varphi$. Then

$$
\begin{aligned}
& \mathbb{P} G^{\prime}=\mathcal{P}^{\prime}(a) \times \mathcal{P}^{\prime}(b) \times \mathcal{P}^{\prime}(c), \\
& \mathbb{P} G=\mathcal{P}^{\prime}(a) \times \mathcal{P}^{\prime}(a) \times \mathcal{P}^{\prime}(b), \\
& \mathbb{P} \varphi(x, y, z)=(x, x, y),
\end{aligned}
$$

and

$$
D \mathbb{P} \varphi(p, q, r)=(p, p, q)
$$

For any $w^{\prime}=\left(w_{a}^{\prime}, w_{b}^{\prime}, w_{c}^{\prime}\right) \in \operatorname{Ctr}\left(G^{\prime}, \mathcal{P}^{\prime}\right)$,

$$
\begin{aligned}
& \left(\mathscr{I}^{\prime}\left(w^{\prime}\right)\right)(x, y, z)=\left(w_{a}^{\prime}(x), w_{b}^{\prime}(x, x, y), w_{c}^{\prime}(y, z)\right), \\
& \varphi^{*} w^{\prime}=\left(w_{a}^{\prime}, w_{a}^{\prime}, w_{b}^{\prime}\right), \\
& \left(\mathscr{I}\left(\varphi^{*} w^{\prime}\right)\right)\left(x_{1}, x_{2}, y\right)=\left(w_{a}^{\prime}\left(x_{1}\right), w_{a}^{\prime}\left(x_{2}\right), w_{b}^{\prime}\left(x_{1}, x_{2}, y\right)\right)
\end{aligned}
$$

and

$$
\left(\mathscr{I}\left(\varphi^{*} w^{\prime}\right) \circ \mathbb{P} \varphi\right)(x, y, z)=\left(w_{a}^{\prime}(x), w_{a}^{\prime}(x), w_{b}^{\prime}(x, x, y)\right)
$$

while

$$
\left(D \mathbb{P} \varphi \circ \mathscr{I}^{\prime}\left(w^{\prime}\right)\right)(x, y, z)=D \mathbb{P} \varphi\left(w_{a}^{\prime}(x), w_{b}^{\prime}(x, x, y), w_{c}^{\prime}(y, z)\right)=\left(w_{a}^{\prime}(x), w_{a}^{\prime}(x), w_{b}^{\prime}(x, x, y)\right)
$$

Hence

$$
\left(\mathscr{I}\left(\varphi^{*} w^{\prime}\right) \circ \mathbb{P} \varphi\right)=\left(D \mathbb{P} \varphi \circ \mathscr{I}^{\prime}\left(w^{\prime}\right)\right)
$$

as expected.

Example 3.15. In Example 3.14 above the map $\varphi: G \rightarrow G^{\prime}$ is neither injective nor surjective. It can, of course, be factored as a surjection $\psi: G \rightarrow G^{\prime \prime}$ :

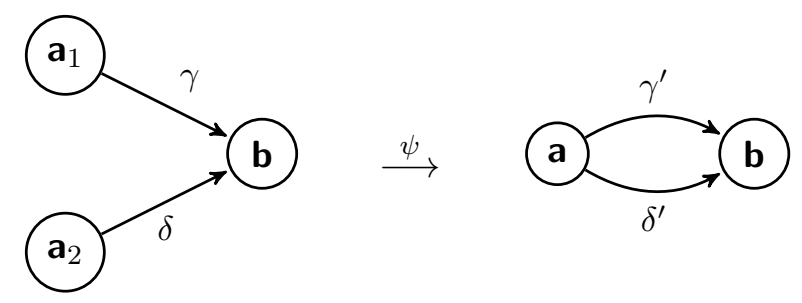


followed by an injection $\iota: G^{\prime \prime} \rightarrow G$ :

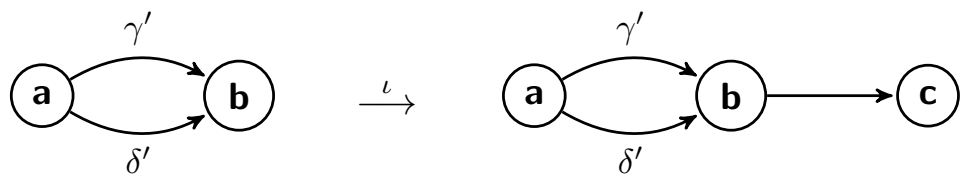

The map $\mathbb{P} \psi: \mathbb{P} G^{\prime \prime} \rightarrow \mathbb{P} G$ is easily seen to be given by

$$
\mathbb{P} \psi(x, y)=(x, x, y)
$$

It is an embedding, as it should be (q.v. Lemma 2.20). The map $\mathbb{P} \imath: \mathbb{P} G^{\prime} \rightarrow \mathbb{P} G^{\prime \prime}$ is given by

$$
\mathbb{P} \imath(x, y, z)=(x, y) .
$$

It is a submersion (q.v. Lemma 2.21). Since $\mathbb{P}$ is a contravariant functor,

$$
\mathbb{P} \varphi=\mathbb{P}(\iota \circ \psi)=\mathbb{P} \psi \circ \mathbb{P} \iota
$$

Theorem 3.11 tells us that for any $w^{\prime}=\left(w_{a}^{\prime}, w_{b}^{\prime}, w_{c}^{\prime}\right) \in \mathcal{C} \operatorname{tr} l\left(G^{\prime}, \mathcal{P}^{\prime}\right)$, the map $\mathbb{P} \imath$ projects the integral curves of the vector field $\mathscr{I}\left(w^{\prime}\right)$ to the integral curves of the vector field $\mathscr{I}\left(\imath^{*} w^{\prime}\right)$ on $\mathbb{P} G^{\prime \prime}$. Furthermore, $\mathbb{P} \psi$ embeds the dynamical system $\left(\mathbb{P} G^{\prime \prime}, \mathscr{I}\left(\imath^{*} w^{\prime}\right)\right)$ into the dynamical system $\left(\mathbb{P} G, \mathscr{I}\left(\varphi^{*} w^{\prime}\right)\right)$. An interested reader can check these two assertions directly.

Example 3.16. Consider the injective graph fibration $\iota: G \rightarrow G^{\prime}$ :

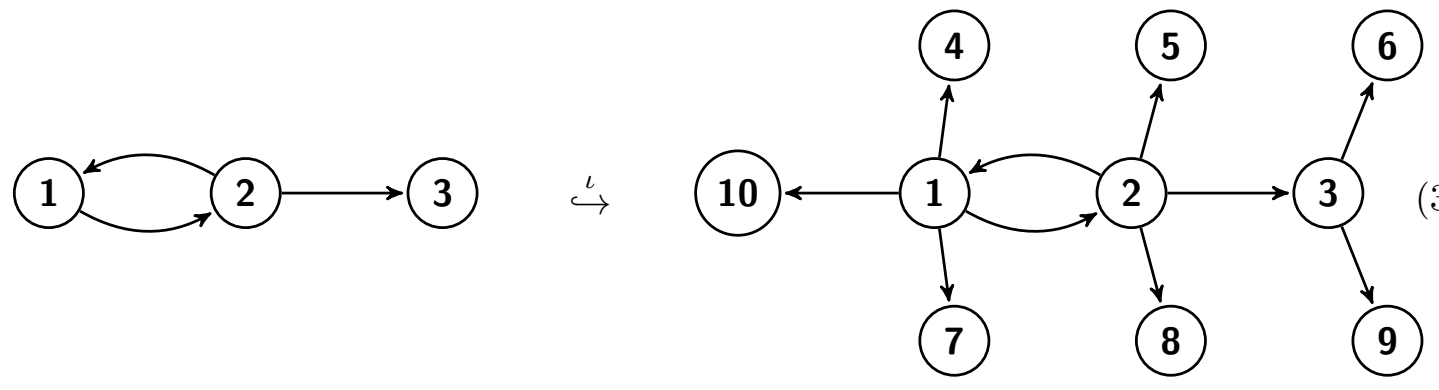

Choose phase space functions $\mathcal{P}, \mathcal{P}^{\prime}$ so that $i:(G, \mathcal{P}) \rightarrow\left(G^{\prime}, \mathcal{P}^{\prime}\right)$ is a map of networks. By Theorem 3.11, for any collection $w^{\prime} \in \operatorname{Ctr}\left[\left(G^{\prime}, \mathcal{P}^{\prime}\right)\right.$ of open systems on the network $\left(G^{\prime}, \mathcal{P}^{\prime}\right)$ the dynamics in the subsystem $\left(\mathbb{P} G, \mathscr{I}\left(i^{*} w^{\prime}\right)\right)$ drives the entire system $\left(\mathbb{P} G^{\prime}, \mathscr{I}\left(w^{\prime}\right)\right)$. This is intuitively clear from the graph (3.4) since there are no "feedbacks" from vertices $4, \ldots, 10$ back into $1,2,3$.

\section{Acknowledgments}

L.D. was supported by the National Science Foundation under grants CMG-0934491 and UBM1129198 and by the National Aeronautics and Space Administration under grant NASA-NNA13 AA91A. The authors also thank the anonymous referees whose comments significantly improved the manuscript.

\section{References}

[1] Awodey S., Category theory, Oxford Logic Guides, Vol. 49, Oxford University Press, New York, 2006.

[2] Boldi P., Vigna S., Fibrations of graphs, Discrete Math. 243 (2002), 21-66. 
[3] Brockett R.W., Control theory and analytical mechanics, in The 1976 Ames Research Center (NASA) Conference on Geometric Control Theory (Moffett Field, Calif., 1976), Lie Groups: History, Frontiers and Appl., Vol. 7, Math Sci Press, Brookline, Mass., 1977, 1-48.

[4] DeVille L., Lerman E., Dynamics on networks I. Combinatorial categories of modular continuous-time systems, arXiv:1008.5359.

[5] DeVille L., Lerman E., Modular dynamical systems on networks, J. Eur. Math. Soc., to appear, arXiv:1303.3907.

[6] Field M., Combinatorial dynamics, Dyn. Syst. 19 (2004), 217-243.

[7] Golubitsky M., Stewart I., Török A., Patterns of synchrony in coupled cell networks with multiple arrows, SIAM J. Appl. Dyn. Syst. 4 (2005), 78-100.

[8] Stewart I., Golubitsky M., Pivato M., Symmetry groupoids and patterns of synchrony in coupled cell networks, SIAM J. Appl. Dyn. Syst. 2 (2003), 609-646.

[9] Tabuada P., Pappas G.J., Quotients of fully nonlinear control systems, SIAM J. Control Optim. 43 (2005), 1844-1866.

[10] Vagner D., Spivak D.I., Lerman E., Algebras of open dynamical systems on the operad of wiring diagrams, arXiv:1408.1598.

[11] Vigna S., The graph-fibrations home page, http://vigna.di.unimi.it/fibrations/.

[12] Willems J.C., On interconnections, control, and feedback, IEEE Trans. Automat. Control 42 (1997), 326339. 